\title{
Selection and its criteria of computational domain on numerical simulation of building fires
}

\author{
Yi Lu ${ }^{1, \mathrm{a},}$ Pan Li ${ }^{1, \mathrm{~b}}$, Jia-Hao Liu ${ }^{1, \mathrm{c},}$ Qin-Pei Chen ${ }^{1, \mathrm{~d}}$, and Jian Wang ${ }^{1, \mathrm{e}, \uparrow}$ \\ ${ }^{1}$ State Key Laboratory of Fire Science, University of Science and Technology of China \\ Hefei, Anhui 230029, People's Republic of China \\ aluyi0813@mail.ustc.edu.cn, ${ }^{b}$ lipan@mail.ustc.edu.cn, ${ }^{1} j$ hliu12@mail.ustc.edu.cn \\ ${ }^{d}$ cqp924@mail.ustc.edu.cn,ewangj@ustc.edu.cn \\ ${ }^{\dagger}$ Corresponding author
}

\begin{abstract}
In the area of numerical simulation of building fires, the computational domain is directly related to the accuracy of the simulation results. This article would be focused on small compartment fires with a single vent opening where heat release rate lied into the transitional region, which is a transformation of the Fuel Controlled Fires into the Ventilation Controlled Fires. The effects of computational domain on simulation results under different room aspect ratios were quantitatively analyzed by correlation analysis. The necessary extension of computational domain beyond physical boundaries of building enclosures was presented in dimensionless form. It provided a theoretical and data reference for the further simulation research in the transitional region.
\end{abstract}

Keywords: Building fires; Numerical simulation; Computational domain; FDS

\section{Introduction}

In the process of numerical simulation, due to the limitation of computer performance, the experimental subjects always need to be simplified, such as geometric model, physic model, boundary conditions, and so on [15]. However, this simplification may reduce the accuracy and validity of the simulation results [11]. Thus, how to select a reasonable and economic computational domain is important. And the simplification of entrainment border [13] becomes one of the chief difficulties. Nevertheless, on account of the lack of the quantitative analysis methods and the complexity of the influence factors [16, 17], previous research principally focused on the qualitative analysis and discussion.

Generally, the building fires can be divided into two kinds of situations. When the burning rate is primarily decided by the fuel properties, this situation is called Fuel Controlled Fires. With the development of fire, when the burning rate is directed by the ventilation conditions, this situation is called Ventilation Controlled Fires. Bjorn Karlsson et al. [18] suggested a formula for the definition of Fuel and Ventilation Controlled Fires: 


$$
\dot{Q}_{v}=1.5 A_{0} \sqrt{H_{0}}(M W)
$$

where $\dot{Q}_{v}$ is the threshold between Fuel and Ventilation Controlled Fires (MW); $A_{0}$ is the area of vent opening (m2); $H_{0}$ is the height of vent opening (m2).

Zhang et al. [19] pointed out that there is a transitional region, in the process from the Fuel Controlled Fires to the Ventilation Controlled Fires. But, the research about transitional region still had gaps. The following research works would present a correlation analysis method. According to this method, the selection criteria of computational domain would be established. Then, the effects of the computational domain extension, under different room sizes, were also investigated.

\section{Numerical Simulation Design}

\section{Geometric model}

The methane burner $(0.27 \times 0.27 \mathrm{~m})$ located at the $0.3 \mathrm{~m}$ height of the room center with constant heat release rates. The burn room dimension was $3.0 \times 3.0 \times 2.28 \mathrm{~m}$ with $0.08 \mathrm{~m}$ thick concrete wall. The size of vent opening was $1.0 \times 1.83 \mathrm{~m}$. The ambient temperature was $20 \mathrm{oC}$. Initial model used the burn room dimension as the computational domain. And the computational domain was extended in the $\mathrm{x}$ - and z-directions only and $\mathrm{Dx}=\mathrm{Dz}$, in the consequent study.

Combining the formula with above parameters, $\dot{Q}_{v}=2748 \mathrm{~kW}$. And the heat release rate (HRR) of the transitional region ranges from $2300 \mathrm{~kW}$ to 3800 $\mathrm{kW}[19]$.

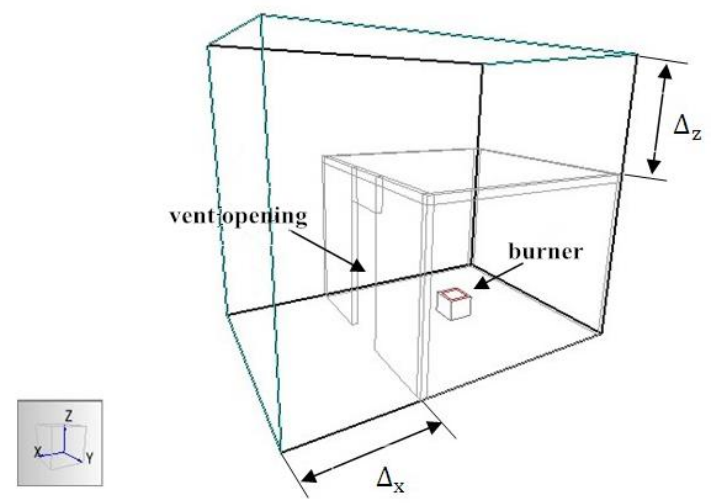

Fig. 1 Schematic diagram of the burn room 


\section{Grid generation}

The gird size of this study was chosen by $D^{*} / \delta x=10_{\text {[23] }}\left(\delta x_{\text {is the grid }}\right.$ size of x-direction. $D^{*}$ is the hydraulic diameter of fire). And $\delta x=\delta y=\delta z$. And the hydraulic diameter of fire was reckoned by the formula of FDS user guide.

\section{Simulation Results}

\section{Analysis of correlation coefficients}

The simulation duration was 150s. In order to eliminate the randomness of instantaneous values, all the quantities presented are the average values from 50 to 150 s. And the subsequent study discussed the effects of the velocity vector with different computational domain extension [23].

In the consequent study, RX\&Y signified the correlation coefficient between the distribution values about velocity vector of case $\mathrm{X}$ and case $\mathrm{Y} . \mathrm{kR}$ signified the absolute values of the derivatives of the correlation coefficients $(R X \& Y)$. And the convergent tendency of RX\&Y would be judged by it $(k R)$.

$$
R_{X \& Y}=\overline{\left(\frac{|Y-X|}{X}\right)}
$$

Where $\mathrm{X}$ and $\mathrm{Y}$ are the sets of variable representing the distribution values of velocity vector of two adjacent cases, the bar above $\mathrm{X}$ and $\mathrm{Y}$ denotes the mean.

\section{Dimensionless processing}

In this article, dimensionless computational domain extension $(\theta)$ was established by the vent opening size.

$$
\theta=\Delta_{x} / D_{H}
$$

Where $\Delta_{x}$ is the computational domain extension of x-direction (m); $D_{H}$ is the hydraulic diameter of vent opening.

The hydraulic diameter is evaluated by

$$
D_{H}=4 A_{0} / P_{0}
$$

Where $A_{0}$ is the area of vent opening (m2); $P_{0}$ is the perimeter of vent opening (m). 
In addition, the dimensionless heat release rate was calculated by formula (5).

$$
Q^{*}=\dot{Q} / \dot{Q}_{v}
$$

\section{Convergence Criteria}

\section{Determining of the convergence criteria}

Using Scene 0 (heat release rate was $3000 \mathrm{~kW}$ ) as an example, grid size was $0.15 \mathrm{~m}$. From Case 0a to $0 \mathrm{~g}$, the extensions were 0.15, 0.30, 0.45, 0.60, 0.75, 0.90 and $1.05 \mathrm{~m}$, respectively. The correlation coefficients Ra\&b, Rb\&c, Rc\&d, Rd\&e, Re\&f and Rf\&g are 0.225, 0.0951, 0.0699, 0.071, 0.041 and 0.0474, respectively. And the corresponding absolute values of the derivatives $(\mathrm{kR})$ are $0.1299,0.07755,0.01205,0.01445,0.0118$ and 0.0064 , respectivley.

According to this method, shown in Fig. 2 and Fig. 3 are the correlation coefficients analysis results of other scenes, whose heat release rate are in the transitional region $(2300 \mathrm{~kW}-3800 \mathrm{~kW})$. In the figures, RX\&Y and $\mathrm{kR}$ decay fluctuantly, and turn to be stable. According to these characteristics, the convergence criteria were determined temporarily, as follows. If the two criteria can be satisfied simultaneously, the computational domain extensions will be considered to be reasonable.
(1) $R_{X \& Y} \leq 0.08$
(2) $k_{R} \leq 0.02$

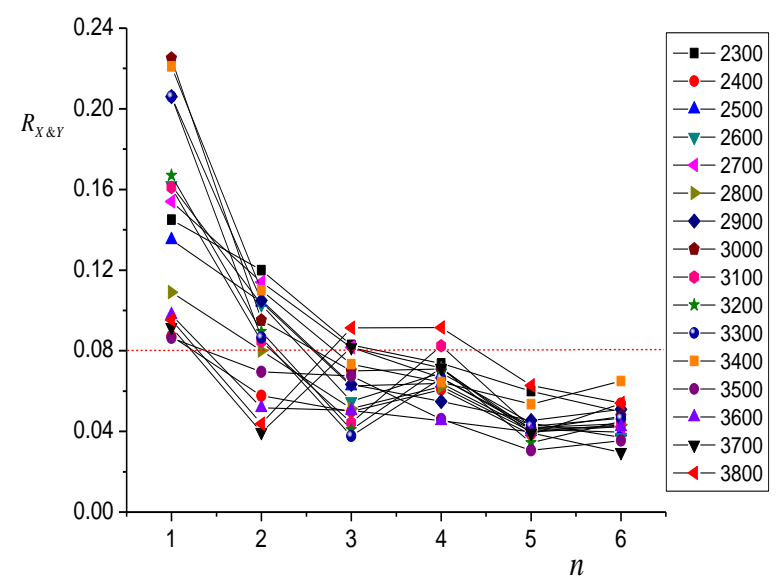

Fig. 2 Correlation coefficients of adjacent domain extensions 


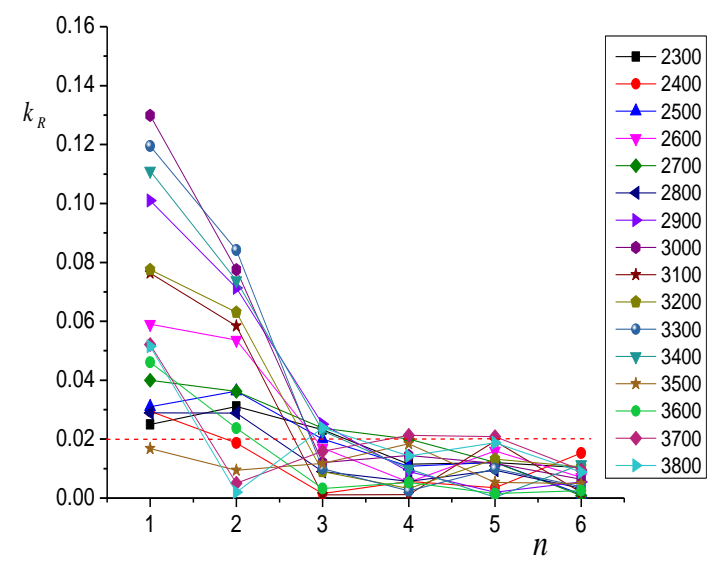

Fig. 3 Absolute values of correlation coefficients derivatives

\section{Verification of the Convergence Criteria}

\section{Dimensionless analysis}

The studies of Zhang [23] and He et al. [14] indicate that dimensionless domain extension

$$
\theta=\left\{\begin{array}{cl}
0.5 & \text { for fuel controlled fire } \\
1 & \text { for ventilation controlled fire }
\end{array}\right.
$$

However, transitional region is a special phase that cannot be distinguished between Fuel and Ventilation Controlled Fires when it begins and ends. This is a coincident situation, combining with the Fuel and Ventilation Controlled Fires. Thus, the results of transitional region maybe cannot conform to the above conclusions accurately. 


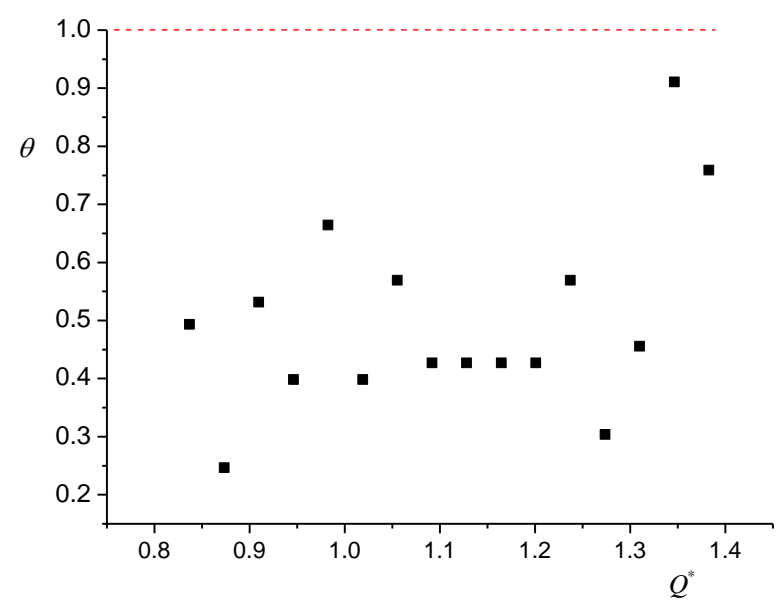

Fig. 4 Scatter diagram of dimensionless heat release rate and dimensionless domain extensions

Yet, Fig. 4 shows that the dimensionless computational domain extensions promote with the growth of the dimensionless heat release rate roughly. Meanwhile, their values vary from 0.4 to 1.0 mostly that is matching to the formula (6). Thus, the convergence criteria were roughly reasonable.

\section{Velocity vector contours analysis}

Using Scene 0 as an example, according to the convergence criteria, the rational computational domain should be $0.45 \mathrm{~m}$. And Fig. 5 shows the velocity vector contours with the different computational domains. With the extension of computational domain, the difference between all the cases is decreasing. When the computational domain extension is $0.45 \mathrm{~m}$ (Case 1c) ,the velocity vector contours of subsequent cases are almost identical. Thus, $0.45 \mathrm{~m}$ can be regarded as the rational selection that is identical to the result of convergence criteria. Therefore, the convergence criteria can be proved to be valid and feasible.

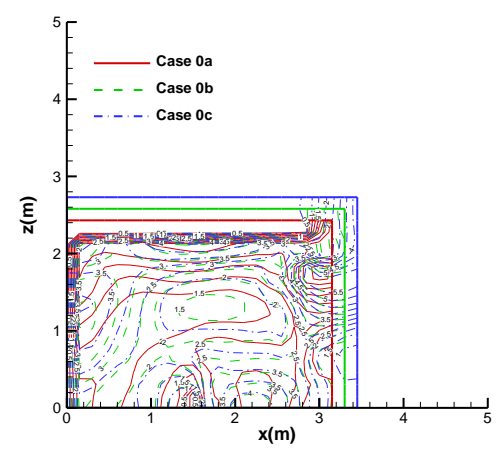

(a)Case 0a, Case 0b, Case0c 


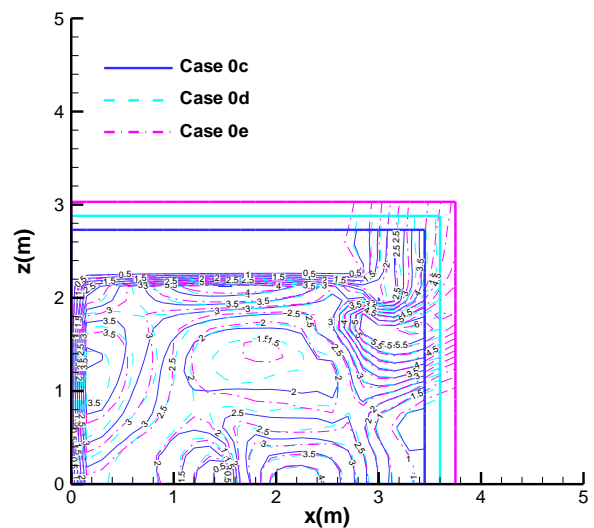

(b) Case0c, Case0d, CaseOe

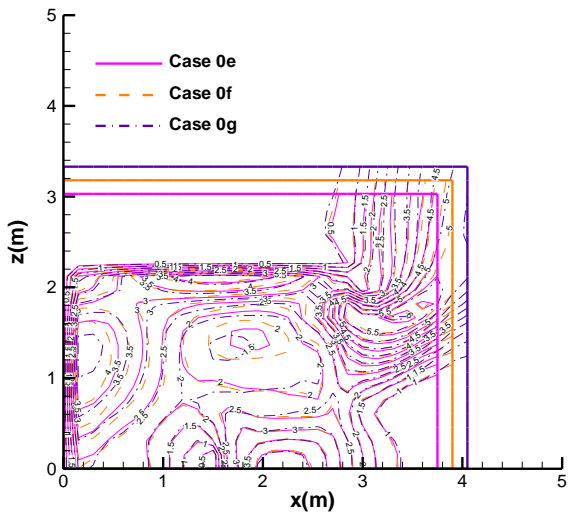

(c) Case0e, Case0f, Case0g

Fig. 5 Comparison of velocity vector contours analysis of Scene $0(\dot{Q}=3000 k W)$

\section{Effects of computational domain selection under different room sizes}

In this subsection, heat release rate was $3000 \mathrm{~kW}$. And grid size was $0.15 \mathrm{~m}$. There were two groups that the room sizes were extended in $\mathrm{x}$ and $\mathrm{y}$ coordinates (Group 1, Group 2), respectively. Afterwards, the optimal values of domain extensions would be analyzed synthetically, combining the velocity vector contours and the convergence criteria. The conclusions were shown in Fig. 6. 


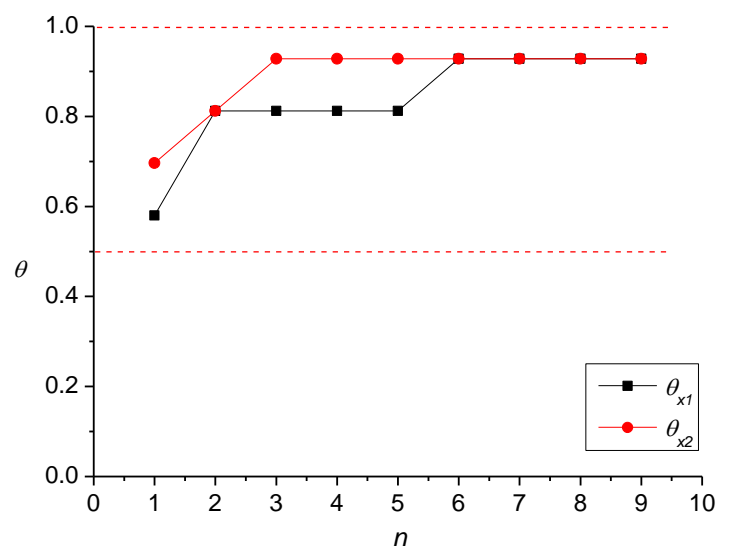

(a) the room sizes were extended in $\mathrm{x}$ coordinate

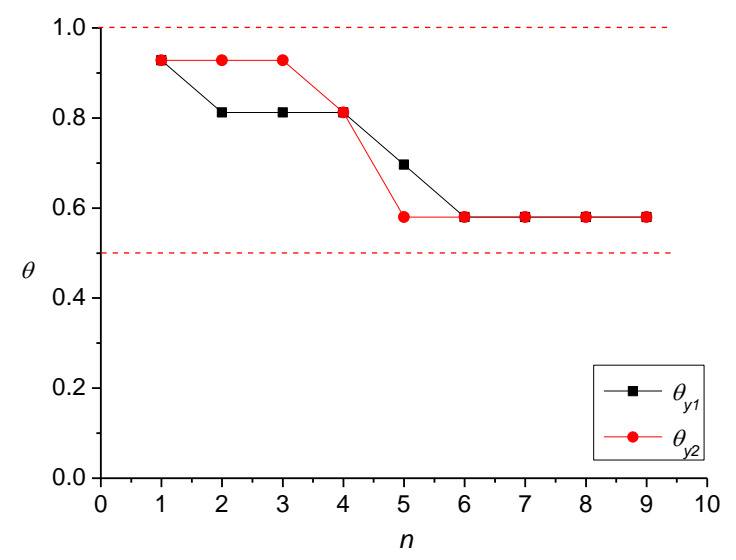

(b) the room sizes were extended in y coordinate

Fig. 6 Dimensionless domain extensions of different room sizes

\section{Dimensionless analysis of computational domain extension}

As shown in Fig. 6 explicitly, the dimensionless computational domain extensions of convergence criteria are a little larger than the ones of the velocity vector contours. This result indicated that the convergence criteria would lead to a more rigorous and reliable conclusion.

In order to strengthen the continuity and correlation of the above conclusions, the room size was transformed into the room aspect ratio $\alpha$. As shown in Fig. 7, the effective domain extension factor varies from 0.5 to 1.0 $(0.5<\theta<1)$, which is consistent with formula (6), basically. 


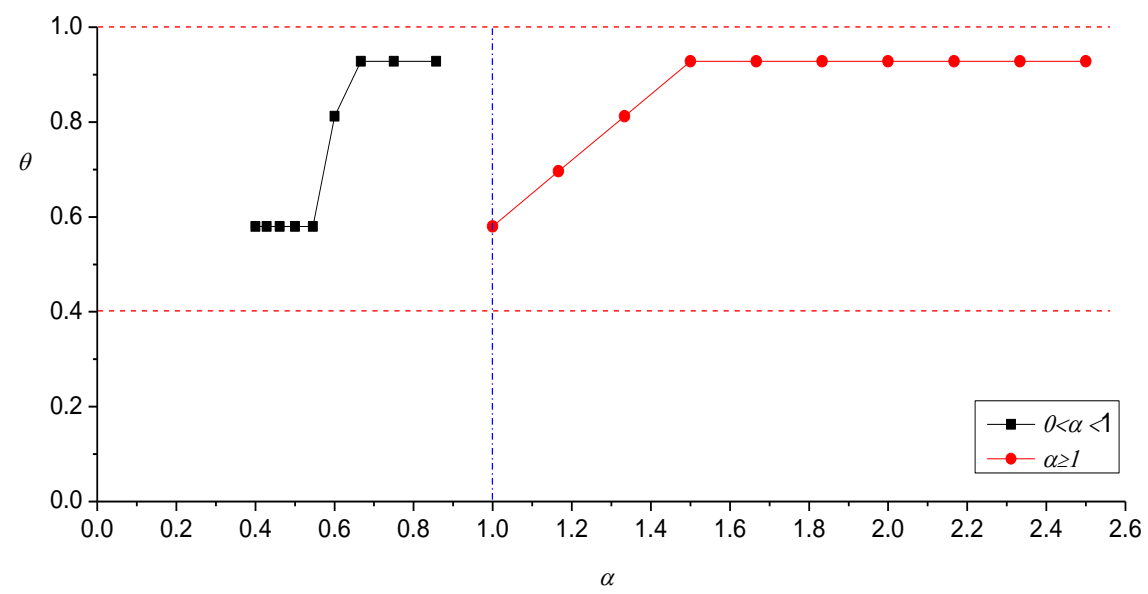

Fig. 7 The dimensionless analysis of domain extension and room aspect ratio

In order to ensure the reliability and applicability of the conclusion, the effective domain extension should be a little larger. Thereby, there was an appropriate selection rule of the effective domain extension about the room aspect ratio in the transitional region:

$$
\theta= \begin{cases}0.6 & 0<\alpha \leq 0.5 \\ 1 & \alpha>0.5\end{cases}
$$

\section{Verification}

Set Scene $19(3300 \mathrm{~kW}, \alpha=1.6667)$ and Scene $20(3200 \mathrm{~kW}, \alpha=0.4000)$ as the verification examples. In Scene 19, the effective domain extensions are $1.0441,0.9281$ respectively, according to the convergence criteria and the velocity vector contours. And in Scene 20, the effective domain extensions are $0.5800,0.5800$ respectively, according to the convergence criteria and the velocity vector contours. These results conform to the selection rules of the previous chapter.

\section{Conclusions}

The main conclusions and innovations were summarized as follows:

(1) Introduced a new correlation analysis to analyze the selection of computational domain quantitatively;

(2) Based on correlation analysis, the selection gist of computational domain, in the transitional region, were 
established as follows: $R_{X \& Y} \leq 0.08, k_{R} \leq 0.02$.

(3) Based on correlation analysis and velocity vector contour analysis, there was the selection rule of the effective domain extension about the room aspect ratio, in the transitional region:

$$
\theta= \begin{cases}0.6 & 0<\alpha \leq 0.5 \\ 1 & \alpha>0.5\end{cases}
$$

\section{References}

[1] Villi G, De Carli M. Detailing the effects of geometry approximation and grid simplification on the capability of a CFD model to address the benchmark test case for flow around a computer simulated person[C]. Building Simulation. Springer Berlin Heidelberg, 2014, 7(1): 35-55.

[2] WU Zhen-kun YANG Man-jiang WANG Jian HE Ya-ping. Mesh Independence in Numerical Simulation of Building Fires [J]. Fire Safety Science and Technology (Chinese), 2005, 24(3): 274-278.

[3] Wu Z K, Yang M J, Wang J, et al. Selection of computational domain in numerical simulation of building fires [J]. Fire Safety Science and Technology, 2006: 232-240.

[4] FU Zhu-man., FAN Wei-cheng., Building fire simulation method and progress [J]. Exploration of Nature (Chinese), 1996, 15(55): 28-33.

[5] ZHU S, HUO R, HU L. Influence of mesh grid and computational domain on FDS simulation [J]. Journal of Safety and Environment, 2008, 4: 033.

[6] KARLSSON B, QUINTIERE J. Enclosure fire dynamics [M]. CRC press, 1999.

[7] ZHANG Xiao-cui., Effects of Computational Domain on Field Modeling of Building Fires [D], Hefei: University of Science and Technology of China, 2010. (in Chinese)

[8] ZHANG Xian-cui, YANG Man-jiang, WANG Jian. Effects of computational domain on numerical simulation of building fires [J]. Journal of Fire Protection Engineering, 2010, 20(4): 225-251.

[9] HE Ya-ping JAMIESON C, JEARY A. Effect of computation domain on simulation of small compartment fires [J]. Fire Safety Science, 2008, 9: 1365-1376. 